\title{
Atividades relacionadas à construção e aprovisionamento de ninhos de Xylocopa subcyanea (Hymenoptera, Apidae) em uma área de restinga na Bahia, Brasil
}

\author{
Miriam Gimenes, Nívia A. Figueiredo \& Andreza H. P. dos Santos
}

Departamento de Ciências Biológicas, Universidade Estadual de Feira de Santana, BR 116, Km 03, 44031-460 Feira de Santana, BA, Brasil.

\begin{abstract}
Activities related to construction and provioning of nests of Xylocopa subcyanea (Hymenoptera, Apidae) in an area of sandbank in Bahia, Brazil. Bees of the genus Xylocopa Latreille, 1802 are frequent in sandbank ecosystems under accelerated degradation. They nest especially in dead or rotted wood. The nest construction and nest structure of Xylocopa (Schoenherria) subcyanea Perez, 1901 were studied in a sandbank from Bahia's north littoral, Brazil. We observed 43 active nests, under different stages of development, in two dead logs. The main activities were excavation of the logs, entering and leaving nest, permanence in the nest entrance, entering with pollen, and nectar dehydration. Preferential times along the day were observed for entering and leaving nest, showing influence of sunrise and sunset times. Abandoned nests were reused by X. subcyanea and Centris tarsata Smith, 1874. Active nests were occupied by four different adult bees. An individual frequently stayed at nest entrance, adopting a defensive behavior. The nest structure was described.
\end{abstract}

KEYWORDS. Apoidea, Xylocopa subcyanea, nest construction, sandbank.

RESUMO. As abelhas do gênero Xylocopa Latreille, 1802 são comuns em ecossistemas de restingas em acelerada fase de degradação e são importantes polinizadores deste ecossistema. Elas nidificam especialmente em madeira morta ou apodrecida. As atividades relacionadas à construção e estrutura dos ninhos de Xylocopa (Schoenherria) subcyanea Perez, 1901 na restinga do litoral norte da Bahia, Brasil, foram observadas em 43 ninhos ativos de X. subcyanea, em diferentes fases de desenvolvimento, em dois troncos apodrecidos. A fase de fundação ou reuso no primeiro tronco foi em agosto e a fase de provisionamento das células nos ninhos, em ambos troncos, em janeiro. As principais atividades foram escavações no tronco, entrada e saída dos ninhos, permanência na entrada do ninho, entrada com pólen e desidratação de néctar. Foi observado horário preferencial ao longo do dia para as atividades de entrada e saída dos ninhos, sendo estas influenciadas pelos horários do nascer e pôr do sol. Ninhos abandonados foram reusados por X. subcyanea e Centris tarsata Smith, 1874. Os ninhos ativos eram ocupados por quatro diferentes abelhas adultas. Geralmente, uma abelha ficava na entrada do ninho. A estrutura do ninho foi descrita.

PALAVRAS-CHAVE. Apoidea, Xylocopa subcyanea, construção de ninhos, restinga.

As abelhas da tribo Xylocopini apresentam distribuição principalmente tropical e subtropical em ambos os hemisférios, sendo bastante diversificadas em regiões áridas neotropicais (MiCHENER, 1979; SILVEIRA et al., 2002). O gênero Xylocopa contém cerca de 750 espécies, sendo 50 registradas no Brasil (HuRd, 1978).

Estudos dos hábitos de nidificação de abelhas do gênero Xylocopa são de grande interesse, considerandose a importância destas abelhas como agentes polinizadores, especialmente de espécies de Passiflora (MANICA, 1981; José et al., 1997; CAmillo, 2003). VARASSIN \& Silva (1999) constataram a importância de Xylocopa brasilianorum (Linnaeus, 1767), Xylocopa frontalis (Olivier, 1789) e Xylocopa ordinaria Smith, 1874 na polinização de Passiflora alata (Passifloraceae) em uma vegetação de restinga em Guarapari, ES. SiHAG (1993), observando a polinização de flores de várias espécies de Cucurbitaceae por Xylocopa fenestrata (Fabricius, 1798), em uma região subtropical na Índia, constatou que a atividade diária da abelha estava sincronizada com os horários de abertura das flores, influenciados pelos valores de temperatura.

$\mathrm{O}$ estudo do potencial polinizador das abelhas do gênero Xylocopa torna-se importante principalmente em ecossistemas frágeis, que sofrem uma acelerada degradação devido à ação antrópica, como nas restingas brasileiras. Reconhecer e compreender a eficiência da polinização, bem como os hábitos e locais de nidificação, podem garantir a sobrevivência dos polinizadores e das espécies vegetais nativas.

De forma geral, estas abelhas nidificam em uma ampla variedade de madeira morta e/ou apodrecida, sendo a arquitetura interna dos ninhos de algumas espécies formada por um sistema de galerias ramificadas, conectadas com o exterior por meio de uma entrada circular ou elíptica (Hurd \& Moure, 1963; SAKAgami \& Laroca, 1971; SAGe, 1968; CAmillo \& Garófalo, 1982, 1989; CAMillo et al., 1986). Em uma área de dunas na Bahia, foi observado que $X$. frontalis, $X$. subcyanea e $X$. cearensis Ducke, 1910 preferem nidificar em galhos secos de Agaristha revoluta (Spr.) DC (Arecaceae) (SILva \& ViAna, 2002; Viana et al., 2002). SiLVEIRA (2002) observou Xylocopa (Stenoxylocopa) artifex Smith, 1874 nidificando em um caule morto de Vellozia sp. em Minas Gerais. Ramalno et al. (2004) registraram a nidificação de Xylocopa (Monoxylocopa) abbreviata Hurd \& Moure, 1963 em caules de Encholirium spectabile Martius ex Schultes f. (Bromeliaceae), planta que ocorre em ambiente de caatinga no semi-árido.

Neste trabalho foram investigadas as atividades, junto à entrada dos ninhos, de fêmeas e machos de $X$. 
subcyanea durante a fundação, construção e aprovisionamento, bem como a estrutura dos ninhos, em duas agregações encontradas na Área de Preservação Ambiental (APA) na restinga de Arembepe, Bahia, Brasil.

\section{MATERIAL E MÉTODOS}

O trabalho foi desenvolvido em um ecossistema de restinga no Sítio Mingu, na Área de Proteção Ambiental (APA) do Rio Capivara, em Arembepe, município de Camaçari, Bahia, Brasil (1243'42'”S - 3808'49' W), nos meses de agosto, outubro, novembro, dezembro de 2001 , janeiro, maio e junho de 2002. O clima de Camaçari é tropical quente e úmido (AM, classificação de Köppen). Durante os meses de estudo, a temperatura média variou entre $21^{\circ}$ e $28^{\circ} \mathrm{C}$ e a umidade relativa de 76,7 a $91,0 \%$. A pluviosidade anual de 2001 foi de $1.528 \mathrm{~mm}$, e a de 2002 de $1.168 \mathrm{~mm}$, sendo o período chuvoso, principalmente, entre março e outubro (dados macroclimáticos obtidos junto à CETREL S.A, Empresa de Proteção Ambiental).

O Sítio Mingu tem aproximadamente 5 ha e caracteriza-se por apresentar uma cobertura vegetal herbácea-arbustiva, composta principalmente por espécies vegetais das famílias Melastomataceae, Scrophulariaceae, Krameriaceae, Leguminosae e Lythraceae, e por um estrato arbóreo-arbustivo composto principalmente por espécies de Vochysiaceae e Malpighiaceae.

As observações foram realizadas de forma ininterrupta das $5 \mathrm{~h}$ às $18 \mathrm{~h}$ (aproximadamente do nascer ao pôr-do-sol), por dois dias consecutivos em cada mês. Foram acompanhadas as atividades externas de fêmeas e machos de $X$. subcyanea presentes nos ninhos localizados em um tronco seco de aproximadamente 1,5 $\mathrm{m}$ de altura e $20 \mathrm{~cm}$ de diâmetro (tronco 1). Neste tronco foram marcados 27 ninhos e as atividades das abelhas monitoradas durante os meses de agosto, outubro, novembro e dezembro de 2001 e janeiro de 2002 . Um segundo tronco (tronco 2) de madeira apodrecida foi encontrado próximo ao tronco 1, onde foram registrados 16 ninhos, acompanhados durante os meses de janeiro, maio e junho de 2002. Foram considerados ninhos recém-fundados ou reutilizados aqueles em que a abertura apresentava-se clara, irregular e com muita serragem na entrada.

Em janeiro de 2002, o tronco 1 foi retirado e devidamente acondicionado em saco plástico e levado para o Laboratório de Entomologia da Universidade Estadual de Feira de Santana. Foi elaborada uma mistura de gesso com tinta solúvel em água e injetada no interior dos ninhos com uma seringa, para a obtenção dos moldes em cores variadas. Após a secagem dos moldes, os ninhos foram abertos.

Dez indivíduos de $X$. subcyanea do tronco 1 foram marcados no tórax, com tinta "guache" atóxica, e liberados no ambiente.

Os dados microclimáticos diários de temperatura, umidade relativa do ar e intensidade luminosa foram coletados em intervalos de uma hora durante todo o estudo de campo. Durante os meses de estudo a temperatura registrada variou entre $23,5^{\circ} \mathrm{C}$ e $33,8^{\circ} \mathrm{C}$ e a umidade relativa de 55 a $93 \%$.

Alguns exemplares de $X$. subcyanea foram coletados e depositados no MZUEFS (Museu de Zoologia da Universidade Estadual de Feira de Santana).
Para verificação do ritmo diário das atividades de entrada e saída, entrada com pólen e de escavação, nos meses de agosto de 2001 e janeiro de 2002, dos 8 ninhos mais ativos, foram utilizados os testes de Rayleigh do método da estatística circular (BATschelet, 1980). Para a obtenção da média e desvio padrão das medidas de largura e comprimento das células, comprimento das galerias e largura da entrada dos ninhos, foi utilizado o programa estatístico "GraphPad Instat".

\section{RESULTADOS E DISCUSSÃO}

No mês de agosto foi observado o maior número de ninhos ativos no tronco $1(n=15)$ (Fig. 1$)$, principalmente em atividade de escavação (ou remoção de serragem) e entrada e saída de indivíduos (Fig. 2), sugerindo que a maior parte destes ninhos estava em fase de fundação ou de reutilização. Nesta atividade a fêmea escavava o ninho com o auxílio de suas mandíbulas. A serragem produzida com esta ação era jogada para fora com a ajuda de suas pernas. Alguns ninhos, porém, encontravam-se apenas com atividade de entrada e saída, como observado no ninho número 5, ocupado por machos e fêmeas. No tronco 2, o maior número de ninhos ativos foi em janeiro $(n=11)$, principalmente em atividade de entrada e saída, entrada com pólen e desidratação de néctar, indicando fase de provisionamento de células. Para a desidratação do néctar, a fêmea ficava parada na entrada do ninho, manipulando o néctar; depois disso ela retornava ao interior do ninho, onde permanecia por alguns minutos e em seguida reaparecia na entrada, reiniciando a manipulação (esse comportamento durava cerca de 15 minutos). O comportamento de desidratação do néctar de $X$. subcyanea é semelhante ao apresentado por Xylocopa grisescens Lepeletier, 1841 e $X$. frontalis (CAmillo \& Garófalo, 1982) e por X. suspecta Moure \& Camargo, 1988 (CAmillo et al., 1986). Segundo esses autores, presume-se que a abelha deposita o néctar na massa de pólen após cada período de desidratação junto à entrada do ninho.

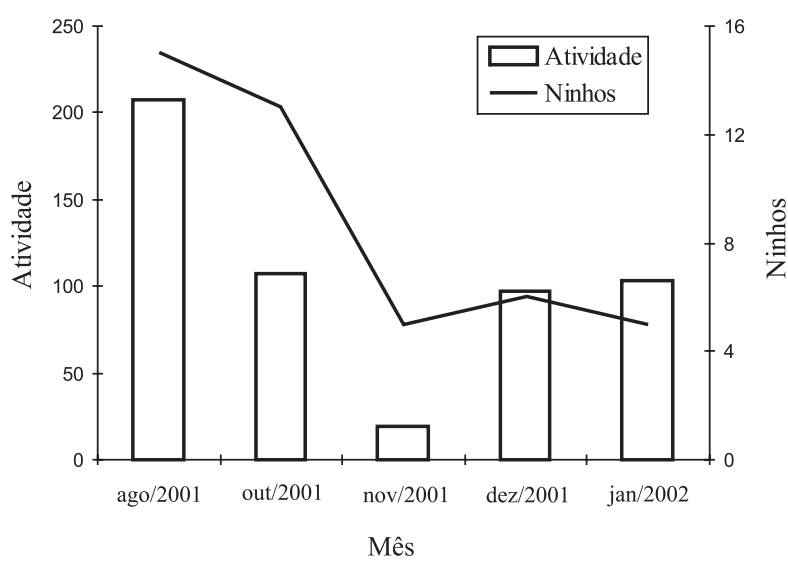

Fig. 1. Atividade de entrada e saída + entrada com pólen + escavação + desidratação e número de ninhos ativos de $X$. subcyanea, nos meses de agosto, outubro, novembro, dezembro de 2001 e janeiro de 2002, em uma área de restinga, Camaçari, BA. 

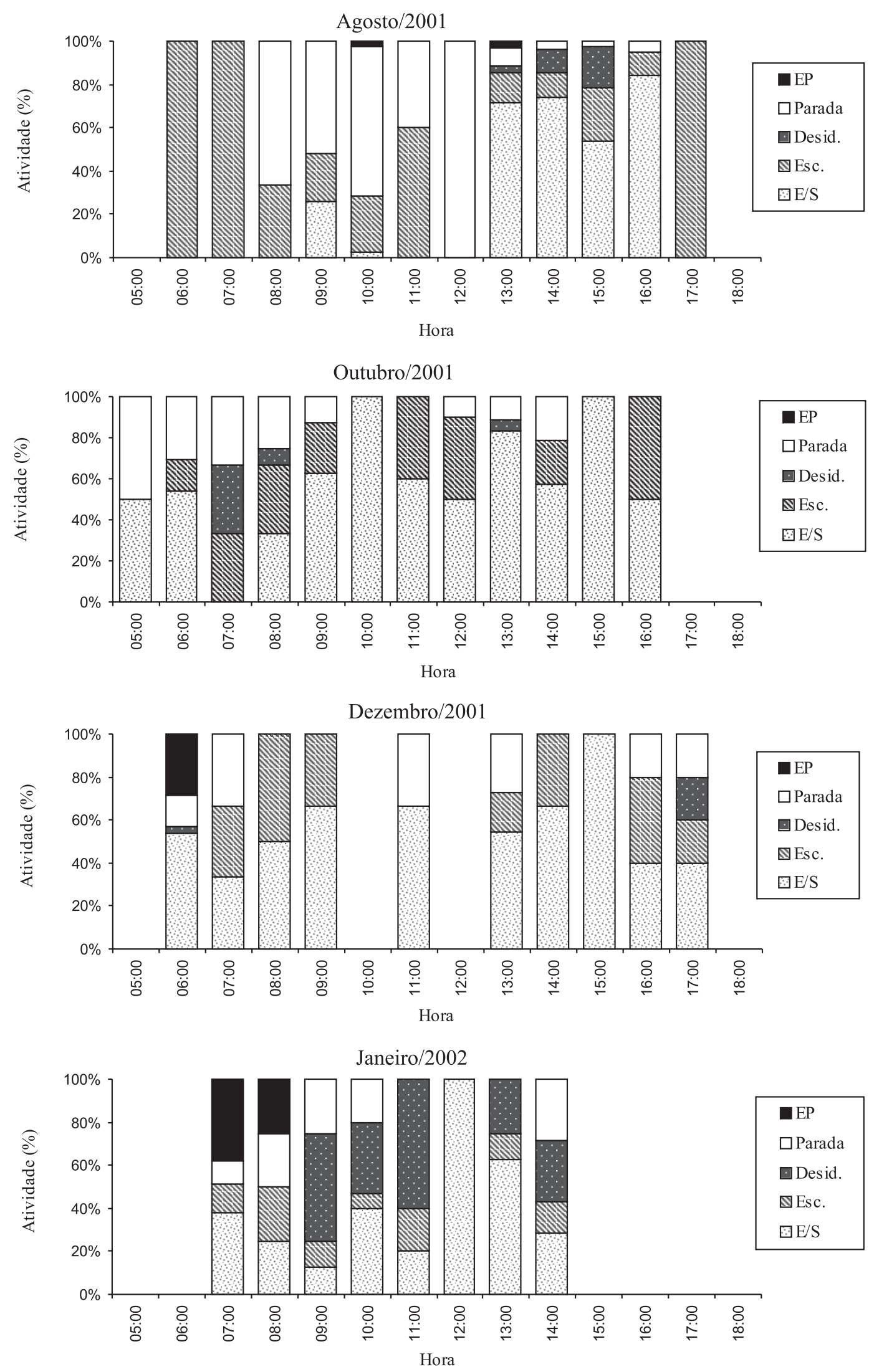

Fig. 2. Atividade dos ninhos de X. subcyanea no tronco 1, nos meses de agosto, outubro, dezembro de 2001 e janeiro de 2002 , em uma área de restinga, Camaçari, BA. (Desid., desidratação de néctar na entrada; EP, entrada com pólen; Esc., escavação do ninho; E/S, entrada e saída de abelhas do ninho; Parada, parada na entrada do ninho). 
Em outubro, observou-se a construção de dois novos ninhos no tronco 1 . Uma das fêmeas iniciou sua escavação e o abandonou logo em seguida, sem ocupação, enquanto que no outro ninho foi registrada atividade até janeiro de 2002. Neste mês também houve predominância das atividades de entrada e saída de abelhas.

Nos meses de novembro, dezembro e janeiro, houve uma queda no número de ninhos ativos $(\mathrm{N}=4 \mathrm{a} 6)$, no tronco 1 (Fig. 1). Em dezembro, observou-se principalmente atividade de entrada e saída de abelhas (Fig. 2). Neste mês, dois ninhos que estavam aparentemente sem atividade foram reutilizados por Centris tarsata Smith, 1874. Estes dois ninhos, quando abertos, apresentavam as paredes revestidas por uma mistura de areia e outra substância não-identificada. Nesses, foram encontradas células com pupas de $C$. tarsata. Outro ninho, também aparentemente sem atividade, foi reutilizado por $X$. subcyanea. A reutilização ou reativação de ninhos por abelhas do gênero Xylocopa também foi observada por outros autores. SAKAGAMI \& LAROCA (1971) observaram reutilização dos ninhos de oito espécies no Sul do Brasil. CAmillo \& Garófalo (1989) observaram a reativação dos ninhos de $X$. suspecta, $X$. frontalis e $X$. grisescens em Ribeirão Preto (SP), e SILVEIRA (2002) observou reutilização de ninhos de Xylocopa (Diaxylocopa) truxali Hurd \& Moure, 1963 e Xylocopa (S.) artifex em galhos de Vellozia (Velloziaceae).

Nos meses de outubro a dezembro, foi registrado o fechamento de muitos ninhos e também a invasão por formigas e cupins. Moscas foram observadas voando próximas aos ninhos, tentando entrar nos mesmos, sendo freqüentemente afastadas pelas abelhas que estavam na entrada.

No tronco 2 o número de ninhos ativos e a freqüência de atividades diminuiu consideravelmente de maio e julho de 2002, sendo que a maior parte dos ninhos encontrava-se com a entrada fechada.

Foi observado, através de abelhas marcadas, que havia quatro indivíduos adultos (machos e fêmeas) residentes no ninho número 26 , e que estes retornavam aos ninhos de origem após as atividades externas. Sempre havia a presença de um indivíduo na entrada dos ninhos. Ao se posicionar somente com a cabeça para fora, a abelha preenchia todo o espaço da entrada, sendo o tamanho da entrada do ninho relativamente proporcional ao da abelha, sugerindo defesa do ninho. Quando alguma abelha tentava entrar em um outro ninho que não o seu, era expulsa com mordidas de mandíbulas pelas residentes. Segundo SAGE (1968), a entrada dos ninhos de Xylocopa fimbriata Fabricius, 1804 apresentou correlação com o tamanho dos indivíduos que os ocupavam, possibilitando uma melhor defesa. Observação similar também foi feita por Viana et al. (2002) para X. cearensis.

Foi observado que as abelhas posicionavam-se com o abdômen na entrada do ninho, eliminando uma substância pastosa de coloração amarelada que pode ser fezes ou outro produto de excreção. A eliminação de substâncias pelo abdômen também foi observada por Kapil \& Dhaliwal (1968), em $X$. fenestrata. Segundo estes autores, $X$. fenestrata, após completar a hibernação, limpava o ninho e descansava a extremidade do abdômen na entrada do mesmo. Durante este processo, ela eliminava uma secreção que poderia servir como repelente para expulsar parasitas e predadores, ou mesmo atribuir um odor específico para o ninho, proporcionando às fêmeas o seu reconhecimento.

Comportamento dos machos. Os machos sobrevoavam a área contendo os ninhos, fazendo uma trajetória em formato de oito sobre as "moitas" de Comolia ovalifolia DC Triana (Melastomataceae), Cuphea brachiata Koehne (Lythraceae) e Borreria verticillata (Linnaeus) GFW Meyer (Rubiaceae), todas freqüentemente visitadas por fêmeas e machos de $X$. subcyanea. Quando algum outro macho tentava se aproximar, era expulso com interações agressivas, sendo perseguido durante o vôo, até que um deles abandonasse a área. Durante o patrulhamento da área, de forma geral, os machos tentavam agarrar as fêmeas que saíam ou que retornavam ao ninho. Em uma destas investidas foi observado que um macho agarrou a fêmea, segurou-a com suas pernas, ficando em posição de cópula. O macho agarrado à fêmea sobrevoou o tronco por alguns segundos, soltando-a em seguida, completando provavelmente a cópula.

$\mathrm{O}$ patrulhamento e as atividades agressivas observadas pelos machos com relação às fêmeas indicam que estes são territoriais, como também observou SAGE (1968) para machos de X. muscaria (Fabricius, 1775) e CAmargo \& Velthuis (1979) para X. frontalis. Machos de X. virginica (Linnaeus, 1771) (Gerling \& Hermann, 1978), X. pubescens Spinola, 1838 (Gerling et al., 1981) e X. californica Cresson, 1864 (Alcock, 1991) também apresentavam o comportamento de patrulhar locais próximos aos ninhos, à procura de fêmeas virgens.

Atividade diária. As atividades externas das fềmeas e dos machos nos ninhos dos dois troncos ocorreram das 5:30 h às 17:00 $\mathrm{h}$, sendo o início das atividades sincronizado com o horário do nascer-do-sol e o término com o pôr-dosol, geralmente com a temperatura acima de $20^{\circ} \mathrm{C}$. A influência da luz do sol também pôde ser observada pela maior concentração de ninhos de $X$. subcyanea nas partes do tronco que eram primeiramente iluminadas, na alvorada. Além disso, as abelhas que estavam nos ninhos que primeiro recebiam a luz do sol saíam antes das abelhas que estavam nos ninhos ainda não-iluminados.

Foi observado que, em agosto de 2001, as atividades de escavação dos ninhos no tronco 1 ocorreram preferencialmente entre $10: 14 \mathrm{~h}$ e $14: 16 \mathrm{~h}$ e para entrada e saída e entrada com pólen ocorreram preferencialmente entre 13:38 he 14:07 h. Para o mês de janeiro, as observações para entrada e saída com pólen dos ninhos do tronco 2 ocorreram preferencialmente entre 7:02 h e 9:08 h e em número maior do que o observado em agosto (Tab. I). Durante os meses observados, considerando todos os ninhos, as atividades de escavação ocorriam em vários horários durante o dia, porém as atividades de entrada com pólen se deram preferencialmente das $6: 00 \mathrm{~h}$ às $8: 00 \mathrm{~h}$ (Fig. 2). O maior número de abelhas ativas ocorreu quando os valores de temperatura estavam entre $26^{\circ}$ e $28^{\circ} \mathrm{C}$ e umidade relativa entre 65 e $66 \%$

As atividades diárias de escavação, entrada e saída e entrada com pólen de mostraram um horário preferencial ao longo do dia, sugerindo um ritmo diário de atividade (Tab. I). Este padrão rítmico variou entre as atividades 
Tabela I. Horários referentes aos ângulos médios, ou horários preferenciais das atividades diárias de entrada com pólen e entrada e saída (EP e ES) e escavação (ESC), nos ninhos mais ativos de $X$. subcyanea dos troncos 1 e 2 , em Arembepe, BA (r, vetor médio ou nível de significância do teste de Rayleigh; N, número de atividade; S, significativo).

\begin{tabular}{|c|c|c|c|c|c|c|c|c|}
\hline Mês & Ninho & Tronco & Ângulo médio (h) & $\mathrm{r}$ & Atividade & Conclusão & $\mathrm{N}$ & $\begin{array}{l}\text { Intervalo de } \\
\text { atividade }(\mathrm{h})\end{array}$ \\
\hline Ago/2001 & 18 & 1 & $13: 38$ & 0,932 & EP e ES & $\mathrm{S}$ & 13 & $10: 00-16: 00$ \\
\hline Ago/2001 & 18 & 1 & $11: 23$ & 0,594 & $\mathrm{ESC}$ & $\mathrm{S}$ & 13 & $6: 00-17: 00$ \\
\hline Ago/2001 & 20 & 1 & $14: 07$ & 0,965 & EP e ES & $\mathrm{S}$ & 15 & $13: 00-16: 00$ \\
\hline Ago/2001 & 20 & 1 & $14: 16$ & 0,81 & ESC & $\mathrm{S}$ & 14 & $10: 00-17: 00$ \\
\hline Ago/2001 & 16 & 1 & $10: 14$ & 0,66 & ESC & $\mathrm{S}$ & 16 & $6: 00-17: 00$ \\
\hline $\mathrm{Jan} / 2002$ & 26 & 1 & $10: 13$ & 0,928 & EP e ES & $\mathrm{S}$ & 8 & $8: 00-13: 00$ \\
\hline $\mathrm{Jan} / 2002$ & 06 & 2 & $7: 02$ & 0,96 & EP e ES & $\mathrm{S}$ & 47 & $6: 00-11: 00$ \\
\hline $\mathrm{Jan} / 2002$ & 10 & 2 & $7: 13$ & 0,944 & EP e ES & $\mathrm{S}$ & 44 & $6: 00-12: 00$ \\
\hline $\mathrm{Jan} / 2002$ & 12 & 2 & $7: 42$ & 0,916 & EP e ES & $\mathrm{S}$ & 78 & $5: 30-14: 00$ \\
\hline $\mathrm{Jan} / 2002$ & 13 & 2 & $9: 08$ & 0,945 & EP e ES & $\mathrm{S}$ & 53 & $6: 00-14: 00$ \\
\hline
\end{tabular}

dos ninhos dos troncos 1 e 2 , provavelmente relacionado às diferentes fases de desenvolvimento em que se encontravam os ninhos estudados, como também observado por CAMILLO et al. (1986) para X. suspecta.

Gerling \& Hermann (1978) observaram que indivíduos de Xylocopa virginica, na Georgia (EUA), iniciavam suas atividades quando a temperatura estava acima de $15^{\circ} \mathrm{C}$, com o pico entre 9:00 h e $11: 00 \mathrm{~h}\left(15-25^{\circ} \mathrm{C}\right)$ e algumas abelhas apresentavam suas últimas coletas no pôr-do-sol. SAGE (1968), estudando abelhas do gênero Xylocopa (Schoenherria) em Guanacaste, província da Costa Rica, observou que $X$. muscaria iniciava suas atividades de coleta nas flores às $5: 45 \mathrm{~h}$, cerca de meia hora antes do nascer-do-sol, e X. subvirescens Cresson, 1879, às 6:35 h. Segundo Gerling \& Hermann (1978), o início das atividades externas destas abelhas pode ser influenciado pela temperatura e o retorno ao ninho influenciado por uma série de fatores, incluindo a luz. Em nosso estudo, porém, observamos que $X$. subcyanea deixava o ninho sempre após o nascer-do-sol, mesmo se antes deste horário a temperatura estivesse acima de $20^{\circ} \mathrm{C}$, sugerindo a influência do nascer e do pôr-do-sol, tanto no início quanto no término das atividades externas. A influência do nascer e pôr-do-sol no ritmo de atividade diária das atividades externas das abelhas já foi observada por GiMENEs et al. $(1993,1996)$.

Estrutura interna dos ninhos de X. subcyanea. O tronco 1 estava bastante apodrecido e com muitos cupins e formigas, apresentando 27 ninhos, sendo cada um deles constituído de um sistema de galerias ramificadas. Cada ninho se ligava ao meio externo através de um orifício de entrada de formato circular, com diâmetro variando de 7 a $8 \mathrm{~mm}(\overline{\mathrm{X}} \pm \mathrm{DP}=7,33 \pm 0,40 \mathrm{~mm}, \mathrm{~N}=6)$. Após a entrada do ninho encontrava-se uma parte mais alargada denominada de vestíbulo, de onde partiam as galerias. $\mathrm{O}$ arranjo de células e galerias observado em $X$. subcyanea é semelhante aos padrões descritos para $X$. frontalis, $X$. grisescens (CAMILlo \& GARÓFALO, 1982; 1989), X. suspecta (Camillo \& Garofalo, 1989; Camillo et al., 1986) e $X$. artifex (Silveira, 2002).

As células consistiam de espaços em formato de barril, separados por partições, de 7,0 a 12,0 $\mathrm{mm}(\overline{\mathrm{X}} \pm \mathrm{DP}$ $=9,0 \pm 1,6 \mathrm{~mm}, \mathrm{~N}=11)$ de comprimento e 5,0 a $8,0 \mathrm{~mm}(5,9$ $\pm 1,0 \mathrm{~mm}, \mathrm{~N}=10$ ) de largura. O comprimento das galerias variou de 44,0 a $91,0 \mathrm{~mm}(65,0 \pm 21,1 \mathrm{~mm}, \mathrm{~N}=5)$.

Ao analisar-se a estrutura interna dos ninhos, não foi verificada comunicação interna entre eles, pois as diferentes cores utilizadas para colorir o gesso em cada ninho não se misturavam, indicando que não havia passagem de um ninho para o outro.

Agregações de ninhos, como observado em $X$. subcyanea, são comuns em abelhas (Michener, 1979), especialmente as pertencentes ao gênero Xylocopa (SAKagami \& Laroca, 1971; Camillo \& Garófalo, 1989). VIANA et al. (2002) observaram semelhante modo de distribuição espacial para X. cearensis nas dunas litorâneas em Abaeté, Salvador, Bahia. Estas agregações podem ser influenciadas, dentre outros fatores, pela quantidade limitada de micro-hábitats, e/ou pela proximidade dos recursos florais (SiHag, 1993; Viana et al., 2002).

Xylocopa subcyanea atua como polinizador eficiente de várias espécies de plantas nativas do ambiente de restinga (Oliveira-Rebouças \& Gimenes, 2004). Neste sentido, obter informações sobre o hábito de nidificação e os horários preferenciais para suas atividades forrageiras e de nidificação são de especial interesse, especialmente, para futuros estudos de conservação da biodiversidade do ecossistema de restinga, que se encontra em acelerado processo de degradação pela ação antrópica.

Agradecimentos. Aos proprietários do Sítio Mingu, por terem permitido o desenvolvimento do trabalho de campo, e à UEFS pelo auxílio financeiro na execução do projeto. Ao M.Sc. Edinaldo L. das Neves (UFBA), pela identificação das abelhas.

\section{REFERÊNCIAS BIBLIOGRÁFICAS}

Alcock, J. 1991. Mate-locating behavior of Xylocopa californica arizonensis Cresson (Hymenoptera: Anthophoridae). Journal of the Kansas Entomological Society 64:349-356.

Batschelet, E. 1980. Circular Statistics in Biology. London, Academic. $371 \mathrm{p}$.

Camargo, J. M. F. \& Velthuis, H. H. W. 1979. Sobre o comportamento de Xylocopa (Megaxylocopa) frontalis (Olivier) (Hymenoptera, Anthophoridae). Dusenia 11: 35-39.

Camillo, E. 2003. Polinização do Maracujá. Ribeirão Preto, Holos. 44p.

Camillo, E. \& Garófalo, C. A. 1982. On the bionomics of Xylocopa frontalis Oliver and Xylocopa grisescens (Lepeletier) in Southern Brazil. I - Nest construction and biological cycle. 
Revista Brasileira de Biologia 42(3):571-582.

1989. Social organization in reactivated nests of three species of Xylocopa (Hymenoptera, Anthophoridae) in southeastern Brazil. Insectes Sociaux 36(2):92-105.

Camillo, E.; Garófalo, C. \& Muccillo, G. 1986. On the bionomics of Xylocopa suspecta (Moure) in Southern Brazil: nest construction and biological cycle (Hymenoptera, Anthophoridae). Revista Brasileira de Biologia 46(2): 383-393.

Gerling, D. \& Hermann, H. R. 1978. Biology and mating behavior of Xylocopa virginica L. (Hymenoptera, Anthophoridae). Behavioral Ecology and Sociobiology 3(2):99-111.

Gerling, D.; Hurd, P. D., JR. \& HefeTz, A. 1981. In-nest behavior of carpenter bee, Xylocopa pubescens Spinola (Hymenoptera: Anthophoridae). Journal of the Kansas Entomological Society 54:209-218.

Gimenes, M.; Benedito-Silva, A. A. \& Marques, M. D. 1993. Chronobiologic aspects of a coadaptive process: The interaction of Ludwigia elegans flowers and its more frequent bee visitors. Chronobiological International 10(1):20-30.

1996. Circadian rhythms of pollen and nectar collection by bees on the flowers of Ludwigia elegans. Biological Rhythm Research 27(3):281-290.

Hurd, P. D. 1978. An annotated catalog of the carpenter bees (genus Xylocopa Latr.) of the Western Hemisphere (Hymenoptera, Anthophoridae). Washington, Smithsonian Institution. $106 \mathrm{p}$.

Hurd, P. D. JR. \& Moure, J. S. 1963. A classification of the large carpenter bees (Xylocopini) (Hymenoptera, Apoidea). University of California Publications in Entomology 29: $1-365$

José, A. R. S.; Bruckner, C. H.; Manica, I. \& Hoffmann, M. 1997. Maracujá: temas selecionados (1). Melhoramento, morte prematura, polinização, taxonomia. Porto Alegre, Cinco Continentes. 70p.

Kapil, R. P. \& Dhaliwal, J. S. 1968. Defense of nest by the female of Xylocopa fenestrata Fab. (Xylocopinae, Hymenoptera). Insects Sociaux 15(4):419-422.

Manica, I. 1981. Fruticultura Tropical. 1. Maracujá. São Paulo, Ceres. $145 \mathrm{p}$.
Michener, C.D. 1979. Biogeography of the bees. Annals of The Missouri Botanical Garden 66: 277-347.

Oliveira-Rebouças, P. \& Gimenes, M. 2004. Abelhas (Apoidea) visitantes de flores de Comolia ovalifolia DC Triana (Melastomataceae) em uma área de restinga na Bahia. Neotropical Entomology 33(3):315-320.

Ramalho, M.; Batista, M. A. \& Silva, M. 2004. Xylocopa (Monoxylocopa) abbreviata Hurd \& Moure (Hymenoptera: Apidae) e Encholirium spectabile (Bromeliaceae): uma associação estreita no semi-árido do Brasil tropical. Neotropical Entomology 33(4):417-425.

SAGE, R. D. 1968. Observations on feeding, nesting, and territorial behavior of carpenter bees genus Xylocopa in Costa Rica. Annals of the Entomological Society of America 61(4):884-889.

SaKagami, S. F. \& Laroca, S. 1971. Observations on the bionomics of some neotropical Xylocopini bees, with some comparative biofaunistics notes (Hymenoptera, Anthophoridae) Journal of the Faculty of Science Hokkaido University 18:57-127.

SinAG, R. C. 1993. Behaviour and ecology of the subtropical carpenter bee, Xylocopa fenestrata F. Journal of Apicultural Research 32(2):64-101.

Silva, F. O. DA \& Viana, B. F. 2002. Distribuição de ninhos de abelhas Xylocopa (Hymenoptera: Apidae) em uma área de dunas litorâneas. Neotropical Entomology 31(4):661-664.

Silveira, F. A. 2002. The bamboo-nesting carpenter bee, Xylocopa (Stenoxylocopa) artifex Smith (Hymenoptera: Apidae), also nesting fibrous branches of Vellozia (Velloziaceae). Lundiana 3(1):57-60.

Silveira, F. A.; Melo, G. A. R. \& Almeida, E. A. B. 2002. Abelhas brasileiras. Sistemática e identificação. Belo Horizonte, Composição \& Arte. 253p.

Varassin, I. G. \& Silva, A. G. DA. 1999. A melitofilia em Passiflora alata Dryander (Passifloraceae), em vegetação de restinga. Rodriguésia 50:5-17.

Viana, B. F.; Kleinert, A. M. P. \& Silva, F. O. 2002. Ecologia de Xylocopa (Neoxylocopa) cearensis (Hymenoptera, Anthophoridae) nas dunas litorâneas de Abaeté, Salvador, Bahia. Iheringia, Série Zoologia, 92(1):47-57.

Recebido em janeiro de 2005. Aceito em maio de 2006. ISSN 0073-4721

Artigo disponível em: www.scielo.br/isz 